\title{
Identical twins with mature cystic teratomas treated with laparoscopic surgery: Two case reports
}

\author{
YASUSHI MABUCHI, NAMI OTA, AYA KOBAYASHI, YUKO TANIZAKI, SAWAKO MINAMI and KAZUHIKO INO \\ Department of Obstetrics and Gynecology, School of Medicine, Wakayama Medical University, Wakayama 641-0012, Japan
}

Received August 5, 2016; Accepted October 20, 2016

DOI: $10.3892 / \mathrm{mco} .2016 .1118$

\begin{abstract}
Mature cystic teratomas are the most common among all ovarian neoplasms, representing 30-40\% of the cases. However, to the best of our knowledge, there have been only two reports of mature cystic teratomas occurring in identical twins to date. We herein report a case of identical twins with mature cystic teratomas who were treated with laparoscopic surgery. A 32-year-old woman was referred to our hospital due to a tumor in the right ovary. The patient underwent laparoscopic resection of the ovarian tumor and the pathological diagnosis was benign mature cystic teratoma. Two years later, the identical twin of the abovementioned patient was referred to our hospital also due to a right ovarian tumor. The patient underwent laparoscopic resection of the ovarian tumor and the pathological diagnosis was benign mature cystic teratoma. Therefore, for early diagnosis, it may be important to consider the possibility of mature cystic teratoma in the identical twin of a patient, even in the absence of symptoms.
\end{abstract}

\section{Introduction}

Mature cystic teratomas are the most common ovarian neoplasms, comprising $30-40 \%$ of the cases. However, to the best of our knowledge, there have been only two reports of mature cystic teratomas occurring in identical twins to date (1). Surgical treatment is required for mature cystic teratoma. Due to frequent occurrence at an early age, laparoscopic resection is often preferred for its reduced invasiveness, particularly in younger women, to preserve the ovary. However, timely access to endoscopic equipment and performing laparoscopic resection may occasionally be challenging in emergent situations, such as torsion or rupture. The incidence of malignant transformation in cases of mature cystic teratoma is reported to be $1-3 \%$ (2), mostly occurring in postmenopausal women. In such cases, oophorec-

Correspondence to: Dr Yasushi Mabuchi, Department of Obstetrics and Gynecology, School of Medicine, Wakayama Medical University, 811-1 Kimiidera, Wakayama 641-0012, Japan

E-mail: booyan@wakayama-med.ac.jp

Key words: ovarian tumor, mature cystic teratoma, laparoscopic surgery, identical twins, familial occurrence tomy or adnexectomy is often performed to avoid recurrence or malignant transformation of the mature cystic teratoma.

For early diagnosis, it may be important to consider the possibility of mature cystic teratoma in the identical twin of a patient, even in the absence of symptoms.

We herein report the case of identical twins with mature cystic teratomas who were treated with laparoscopic surgery.

\section{Case presentation}

Case 1. In August, 2012, a 32-year-old woman visited a gynecologist and requested cancer screening, without any symptoms. Pelvic examination and ultrasonography revealed the presence of an ovarian tumor and the patient was referred to our Department of Obstetrics and Gynecology (Wakayama Medical University, Wakayama, Japan). Magnetic resonance imaging revealed a cystic tumor sized $7 \times 9 \mathrm{~cm}$ in the right adnexa (Fig. 1A and B). The patient underwent laparoscopic resection of the tumor. On macroscopic examination, the tumor was found to contain adipose tissue and hair, and the pathological diagnosis following histological evaluation was benign mature cystic teratoma (Fig. 1C). The postoperative course was uneventful.

Case 2. Approximately 2 years later (February, 2014) a 34-year-old woman, the identical twin of the patient in case 1, visited a gynecologist wishing to conceive. Pelvic examination and ultrasonography revealed the presence of an ovarian tumor and the patient was referred to our hospital. On magnetic resonance imaging, a cystic tumor sized $7 \times 8 \mathrm{~cm}$ was identified in the right adnexa (Fig. 2A and B). The patient underwent laparoscopic resection of the right ovarian tumor. On macroscopic examination, this tumor also contained adipose tissue and hair and the pathological diagnosis was benign mature cystic teratoma (Fig. 2C). The postoperative course of the patient was uneventful.

Written informed consent to the publication of the case details was obtained from the two patients, and patient anonymity was preserved.

\section{Discussion}

To the best of our knowledge, there have been only two reports of identical twins with mature cystic teratomas to date. Brown et al reported the case of a 24 -year-old patient with a 

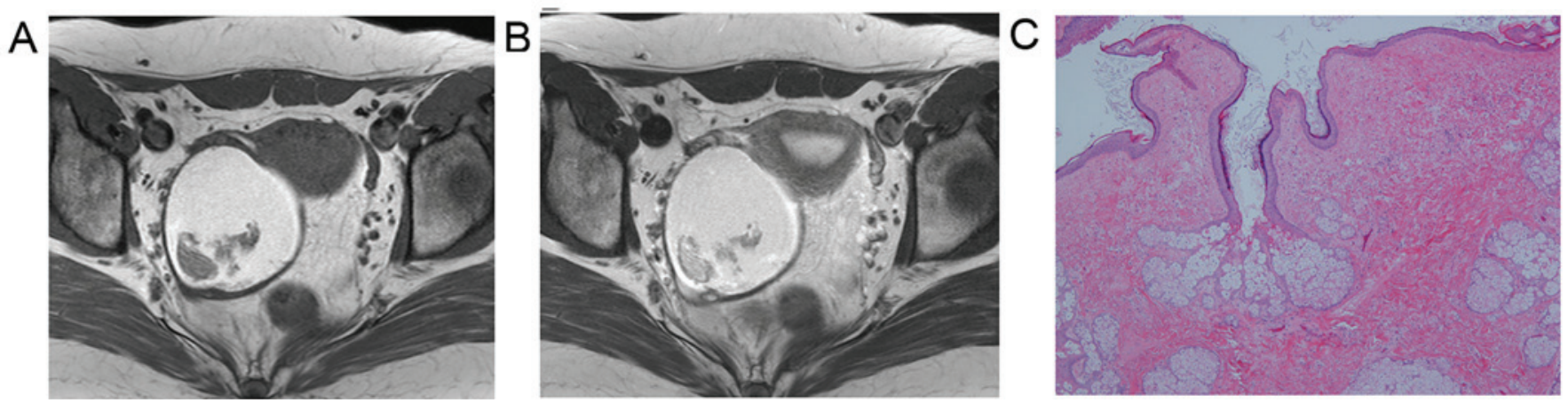

Figure 1. (A and B) Magnetic resonance imaging findings in case 1; (A) T1- and (B) T2-weighted images revealed a cystic tumor, sized 7x9 $\mathrm{cm}$, in the right adnexa. (C) Histological findings in case 1. Squamous epithelia and sebaceous glands were observed (hematoxylin and eosin staining; magnification, $\mathrm{x} 40$ ).
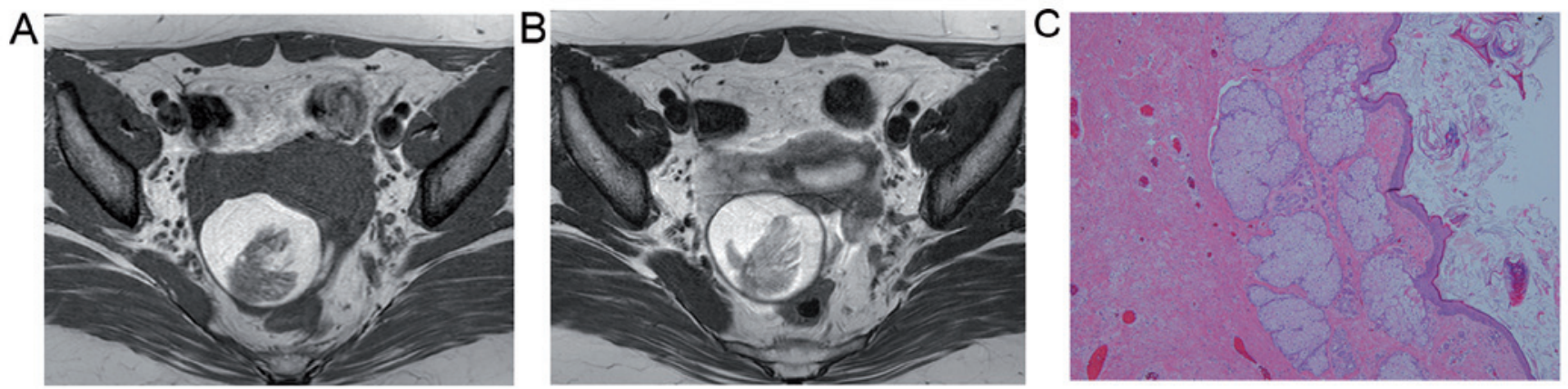

Figure 2. (A and B) Magnetic resonance imaging findings in case 2. (A) T1- and (B) T2-weighted images revealed a cystic tumor, sized 7x8 cm, in the right adnexa. (C) Histological findings in case 2. Squamous epithelia and sebaceous glands were observed (hematoxylin and eosin staining; magnification, x40).

twisted benign cystic teratoma of the right ovary and a benign cystic teratoma of the left ovary (1); they also reported the same twisted benign cystic teratoma of the right ovary and benign cystic teratoma of the left ovary in the identical twin sister of the abovementioned patient, who was treated 2 years later (1). Laparotomy with removal of the right adnexa and left ovarian cystectomy were performed in both cases (1). Simon et al reported identical twins with twisted mature cystic teratoma of the right ovary (3). The diagnoses were confirmed at laparotomy and subsequent histopathological examination $(1,3)$.

Feld et al reported the removal of bilateral ovarian benign cystic teratomas from a set of probable identical triplets (4). In addition, Indinnimeo et al reported heterozygotic twins with benign cystic teratomas of the ovary (5) and Brenner et al reported the familial occurrence of benign cystic teratoma in three successive generations (6): A 19-year-old woman, her 45-year-old mother and her 78-year-old grandmother underwent surgery due to ovarian tumors, and the pathological diagnosis was benign cystic teratoma in all cases (6). Plattner et al also reported the familial incidence of bilateral ovarian dermoid cysts in a mother and her two daughters (7).

In the present case, the identical twins had ovarian tumors that were similar in size, and were both affected on the same side at approximately the same age. Laparoscopic surgery and pathological examination confirmed the diagnosis of mature cystic teratoma in both cases. The postoperative courses were uneventful and normal menstrual cycles resumed after surgery.

Linder et al suggested that benign cystic teratoma of the ovary may be a parthenogenetic tumor arising from a single germ cell after the first meiotic division (8). Hecht et al suggested the existence of genes in humans that dictate the formation and fate of the second polar body in meiosis, and that mutations of these genes may disturb this normal process and predispose females to develop ovarian teratomas (9). It is reported that ovarian teratomas usually occur during the reproductive age, and mature cystic teratomas have a high reported incidence of bilaterality (15-18\%) (10). Early onset and bilaterality are common characteristics of hereditary tumors (11). However, there is no evidence that mature cystic teratomas are a congenital disease due to a genetic disorder. The rate of monozygotic twins in Japan was 3.0 per 1,000 births in 1985 (12), whereas the respective rates in the United States were reported to be 4.7 (African American) and 4.2 (Caucasian) per 1,000 births in 1985 (12). Mature cystic teratomas account for $30-40 \%$ of all ovarian neoplasms. The rarity of identical twins with mature cystic teratomas is interesting, considering the prevalence of mature cystic teratoma and identical twins; thus, it is possible that there may be more unreported cases of identical twins with this type of tumor.

Surgical treatment is required for mature cystic teratoma. Due to frequent occurrence at an early age, laparoscopic resection is often preferred for its reduced invasiveness, particularly in younger women, for preservation of the ovary. However, timely access to endoscopic equipment and performing laparoscopic resection may occasionally be challenging in emergent situations, such as torsion or rupture. The incidence of malignant transformation in cases of mature cystic teratoma is reported to be $1-3 \%$ (2), mostly occurring in postmenopausal women. In such cases, oophorectomy or adnexectomy is often performed to avoid recurrence or malignant transformation of the mature cystic teratoma. 
Thus, for early diagnosis, it may be important to consider the possibility of mature cystic teratoma in the identical twin of a patient, even in the absence of symptoms. It is preferable that the identical twin of a patient is informed on the possibility of an ovarian tumor, including mature cystic teratoma, even if she is asymptomatic; visiting a gynecologist to check the ovaries is recommended in the presence of any symptoms, such as abdominal pain or distension.

\section{References}

1. Brown EH Jr: Identical twins with twisted benign cystic teratoma of the ovary. Am J Obstet Gyneccol 134: 879-880, 1979.

2. Curling OM, Potsides PN and Hudson CN: Malignant change in benign cystic teratoma of the ovary. Br J Obstet Gynecol 86: 399-402, 1979.

3. Simon A, Ohel G, Neri A and Schenker G: Familial occurrence of mature cystic teratomas. Obstet Gynecol 66: 278-279, 1985.

4. Feld D, Labes J and Nathanson M: Bilateral ovarian dermoid cysts in triplets. Obstet Gynecol 27: 525-528, 1966.
5. Indinnimeo M, Cicchini C, Laricinese A, Kanakaki S, Ricci F and Mingazzini PL: Two twins with teratoma of the ovary. An unusual association: Case report. Eur J Gynaecol Oncol 24: 199-201, 2003.

6. Brenner SH and Wallach RC: Familial benign cystic teratoma. Int J Gynaecol Obstet 21: 167-169, 1983.

7. Plattner G and Oxorn H: Familial incidence of ovarian dermoid cysts. Can Med Assoc J 108: 892-893, 1973.

8. Linder D, McCaw BK and Heckt F: Parthenogenic origin of benign ovarian teratomas. N Engl J Med 292: 63-66, 1975.

9. Hecht F, McCaw BK and Patil S: Ovarian teratomas and genetics of germ-cell formation. Lancet 2: 1311, 1976.

10. Novak E and Woodruff J: Gynecologic and Obstetric Pathology. W. B. Saunders Company, Philadelphia, PA, 485-490, 1979.

11. Knudson AG Jr, Strong LC and Anderson DE: Heredity and cancer in man. Prog Med Genet 9: 113-158, 1973.

12. Cunningham F, Leveno K, Bloom S, et al: Williams Obstetrics. 24th edition. McGraw Hill Education, 2014. 